Fourier reconstruction of functions

from their nonstandard sampled Radon transform

Daniel Potts and Gabriele Steidl

Nr. 258

January 2001 


\title{
Fourier reconstruction of functions from their nonstandard sampled Radon transform
}

\author{
Daniel Potts \\ Medical University of Lübeck \\ Institute of Mathematics \\ D-23560 Lübeck \\ Germany \\ potts@math.mu-luebeck.de
}

\author{
Gabriele Steidl: \\ University of Mannheim \\ Institute of Computer Science \\ D-68131 Mannheim \\ Germany \\ steidl@math.uni-mannheim.de
}

\begin{abstract}
In this paper, we suggest a new Fourier transform based algorithm for the reconstruction of functions from their nonstandard sampled Radon transform. The algorithm incorporates recently developed fast Fourier transforms for nonequispaced data. We estimate the corresponding aliasing error in dependence on the sampling geometry of the Radon transform and confirm our theoretical results by numerical examples.
\end{abstract}

\section{Mathematics Subject Classification. 44A12, 65T50}

Key words and phrases. Fast Fourier transform for nonequispaced data, Radon transform, computerized tomography, gridding, interlaced grid, nonstandard sampling, aliasing error

\section{Introduction}

Let $\mathcal{S}\left(\mathbb{R}^{2}\right)$ be the Schwartz space of rapidly decreasing functions and let $\mathcal{S}(\mathbb{R} \times \mathbb{T})$ be the space of $C^{\infty}$ functions on $\mathbb{R}^{2}$ that are rapidly decreasing in the first argument and $2 \pi$-periodic in the second argument.

We are interested in the two-dimensional computerized parallel beam tomography which is conveniently modeled by the Radon transform $R: \mathcal{S}\left(\mathbb{R}^{2}\right) \rightarrow \mathcal{S}(\mathbb{R} \times \mathbb{T})$ with

$$
R f(s, \varphi):=\int_{x \theta=s} f(x) \mathrm{d} x \quad\left(\theta:=\left(\begin{array}{c}
\cos \varphi \\
\sin \varphi
\end{array}\right)\right)
$$

The line integrals are parameterized by the angle $\varphi \in \mathbb{T}$ between the beam normal vector and the abzissa and by the distance $s \in \mathbb{R}$ of the beam from the origin. See Figure 1 .

In general, we assume that $f$ has compact support, i.e. without loss of generality that

$$
\operatorname{supp} f \subseteq \Omega:=\left\{x \in \mathbb{R}^{2}:\|x\| \leq 1\right\}
$$

By $C_{0}^{\infty}(\Omega)$ we denote the space of $C^{\infty}$ functions with compact support in $\Omega$. Let

$$
\hat{f}(\xi):=\int_{\mathbb{R}^{n}} f(x) \mathrm{e}^{-2 \pi \mathrm{i} x \xi} \mathrm{d} x
$$

be the Fourier transform of $f \in L_{2}\left(\mathbb{R}^{n}\right)$. 


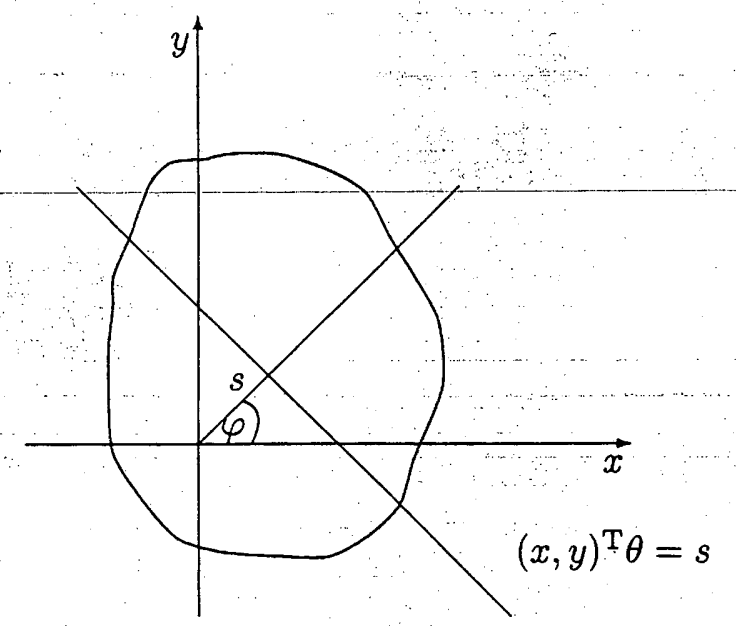

Figure 1: $\mathrm{X}$-ray in parallel beam tomography

Radon transform and Fourier transform are related by the so-called "Fourier slice theorem" or "projection theorem".

Theorem 1.1 If $f \in \mathcal{S}\left(\mathbb{R}^{2}\right)$, then

$$
\hat{f}(\sigma \theta)=\int_{\mathbb{R}} R f(s, \varphi) \mathrm{e}^{-2 \pi i s \sigma} \mathrm{d} s=\widehat{R f}(\sigma, \varphi) .
$$

In practice only a finite number of line integrals (1.1) can be measured. So the natural question arises which line integrals should be taken, i.e. how the beams should be arranged in order to insure a satisfactory reconstruction of the function $f$.

If we sample $R f$ on a grid $\mathcal{G}:=\left\{\boldsymbol{A} k: k \in \mathbb{Z}^{2}\right\} \subseteq \mathbb{R} \times \mathbb{T}$, the answer was given by Natterer $[20]:$

Let $\hat{\mathcal{G}}:=\left\{\hat{A} k: k \in \mathbb{Z}^{2}\right\} \subseteq \mathbb{R} \times \mathbb{Z}$ with a nonsingular generating matrix

$$
\hat{\boldsymbol{A}}:=\left(\begin{array}{ll}
a_{11} & a_{12} \\
a_{21} & a_{22}
\end{array}\right) \in \mathbb{R}^{2 \times 2} ;\left(a_{21}, a_{22} \in \mathbb{Z}\right)
$$

denote the dual grid of the sampling grid $G:=\left\{\boldsymbol{A} k: k \in \mathbb{Z}^{2}\right\} \subseteq \mathbb{R} \times \mathbb{T}$, where

$$
\boldsymbol{A}:=\left(\begin{array}{r}
1,0 \\
0,2 \pi
\end{array}\right)\left(\hat{\boldsymbol{A}}^{-1}\right)^{\mathrm{T}}=\frac{1}{\operatorname{det}(\hat{\boldsymbol{A}})}\left(\begin{array}{c}
a_{22}-a_{21} \\
-2 \pi a_{12} 2 \pi a_{11}
\end{array}\right)
$$

Theorem 1:2 [20, Theorem III.3.1] Let $f \in C_{0}^{\infty}(\Omega)$ and $R f \in \mathcal{S}(\mathbb{R} \times \mathbb{T})$. For $\nu \in(0,1)$ and $b \geq 1$ define the set $K$ by

$$
K:=\left\{(\sigma, k) \in \mathbb{R} \times \mathbb{Z}:|\sigma|<b,|k|<2 \pi \max \left\{\frac{|\sigma|}{\nu},\left(\frac{1}{\nu}-1\right) b\right\}\right\}
$$

Let $\hat{\boldsymbol{A}}$ be given by (1.3) so that 
(A) the sets $K+\hat{A} k\left(k \in \mathbb{Z}^{2}\right)$ are mutually disjoint.

If $R f(A k)=0$ for all $k \in \mathbb{Z}^{2}$, then we have for $b \geq B(\nu) \geq 1$ that

$$
\|R f\|_{L_{\infty}(\mathbb{R} \times \mathbb{T})} \leq C(\nu) \mathrm{e}^{-\lambda(\nu) b}\|f\|_{L_{1}(\Omega)}+\frac{8}{\pi \nu} \varepsilon_{0}(f, b)
$$

Here $C(\nu)$ and $\lambda(\nu)$ are positive constants and

$$
\varepsilon_{0}(f, b):=\int_{|\xi| \geq b}|\hat{f}(\xi)| \mathrm{d} \xi
$$

The first summand in (1.5) decays exponentially as $b$ increases beyond $B(\nu)$, while the second summand depends on the decay of $|\hat{f}|$ outside the ball $\{\xi:|\xi| \leq b\}$.

The simplest sampling grid $\mathcal{G}$ which satisfies the assumptions of Theorem 1.2 is the so-called standard grid with generating matrix

$$
A=\left(\begin{array}{cc}
\frac{1}{2 b} & 0 \\
0 & \frac{\pi}{T}
\end{array}\right) \quad(T>2 b \pi)
$$

Here

$$
\hat{\boldsymbol{A}}=\left(\begin{array}{cc}
2 b & 0 \\
0 & 2 T
\end{array}\right)
$$

generates the dual grid. This is the grid usually applied in parallel beam computerized tomography and the assumption $(\mathcal{A})$ in Theorem 1.2 is simply an agreement with a result of Bracewell and Riddle [3]. See Figure 2. The standard grid has mesh width $\leq \frac{1}{4 b^{2}}$. We will refer to any other grid $\mathcal{G}$ as nonstandard grid. Having a look at Figure 2, we see that $\hat{\mathcal{G}}$ can be further refined so that $\mathcal{G}$ becomes coarser.

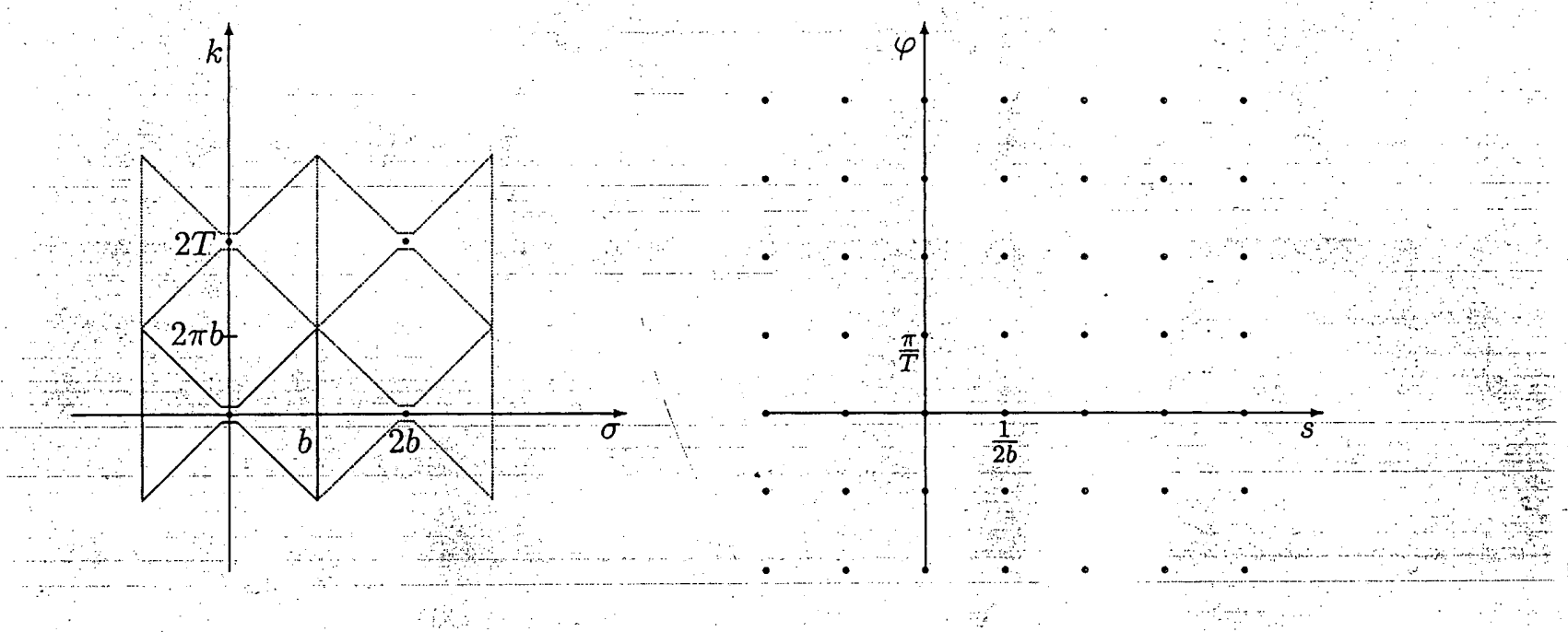

Figure 2: Dual standard grid with four sets $K+\hat{\boldsymbol{A}} k$ (left) and standard grid (right), where $T=\frac{2 \pi b}{\nu}(\nu \approx 0.95)$. 
For example, the sampling grid and corresponding dual grid with generating matrices

$$
\boldsymbol{A}:=\left(\begin{array}{cc}
\frac{1}{b} & \frac{1}{2 b} \\
0 & \frac{\pi}{T}
\end{array}\right), \hat{A}=\left(\begin{array}{cc}
b & 0 \\
-T & 2 T
\end{array}\right) \quad(T>2 b \pi)
$$

respectively, also fulfill the assumption of Theorem 1.2. See Figure 3.

Moreover, this so-called interlaced grid has twice the mesh width of the standard grid, i.e. we need only half the number of line integrals for a satisfactory reconstruction of $f$ than in the standard sampling case. The interlaced grid has been found by Lindgren and Rattey [18], while its name is due to Cormack [4]:

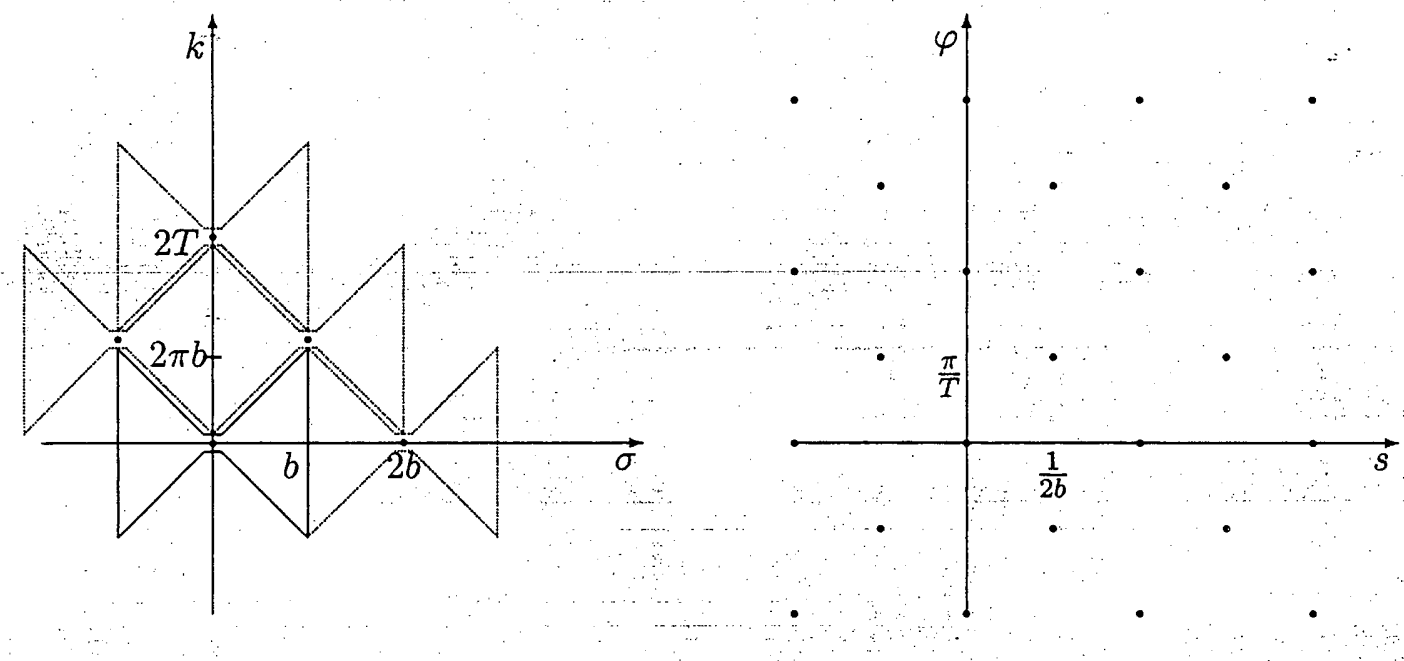

Figure 3: Dual interlaced grid with four sets $K+\hat{A} k$ (left) and interlaced grid (right), where $T=\frac{2 \pi b}{\mu}(\mu<\nu ; \nu \approx 0.95)$.

Various algorithms were designed for the reconstruction of functions $f$ from their sampled values on the interlaced grid. For example, a filtered backprojection algorithm was suggested by Kruse [15] and algebraic reconstruction algorithms by Klaverkamp [14].

In this paper, we are interested in Fourier reconstruction algorithms based on the projection theorem 1.1. Fourier reconstruction algorithms for standard sampled Radon transforms have recently attained new attention in connection with fast Fourier transform algorithms for nonequispaced data $[10,31,11,25]$. These new algorithms improve known Fourier reconstruction algorithms as the UFR/gridding algorithm $[16,17,22,26]$ or the linogram algorithm $[7,-27]$ with respect to the reconstruction speed and-image quality. In contrast to the convenient filtered backprojection which ensures a good quality of the image at the expense of $O\left(N^{3}\right)$ arithmetic operations, Fourier reconstruction methods require only $\mathcal{O}\left(N^{2} \log N\right)$ arithmetic operations. This feature will be of particular interest for future three-dimensional image processing.

This paper is organized as follows: The following section provides a new Fourier reconstruction algorithm for nonstandard sampled-Radon transforms based on fast Fourier transforms 
for nonequispaced data. Section 3 contains estimates of the aliasing errors introduced by our algorithm which confirm Natterer's sampling condition $(\mathcal{A})$. Finally, Section 4 presents numerical results.

\section{Fourier reconstruction algorithms}

Our Fourier reconstruction algorithms are based on fast Fourier transforms for nonequispaced data (NFFT). In the following, we introduce, the NFFT in a very short way. For details see [24].

Let $\Pi^{d}:=\left[-\frac{1}{2}, \frac{1}{2}\right)^{d}$ and $I_{N}:=\left\{k \in \mathbb{Z}^{d}:-\frac{N}{2} \leq k<\frac{N}{2}\right\}$, where the inequalities hold componentwise. For arbitrary $w_{j} \in \Pi^{d}\left(j \in I_{M}\right)$, we consider

$$
\hat{f}_{k}:=\sum_{j \in I_{M}} f_{j} \mathrm{e}^{-2 \pi \mathrm{i} k w_{j}} \quad\left(k \in I_{N}\right)
$$

Straightforward computation of (2.1) requires $\mathcal{O}\left((M N)^{d}\right)$ arithmetic operations. For equispaced data $w_{j}:=j / N\left(j \in I_{N}\right)$ the complexity can be reduced to $\mathcal{O}\left(N^{d} \log N\right)$ arithmetic operations by applying well-known fast Fourier transform techniques (FFT).

Recently, fast approximative algorithms for the efficient computation of (2.1) were developed $[2,6,23,29,32]$. The basic idea of these algorithms was known as so-called "gridding". However, only the recent papers clarify the relation between the speed of the algorithm and the introduced approximation error and give non-heuristic criteria for the choice of the parameters of the algorithm.

Let $n:=\alpha N \quad(\alpha>1)$ and $m \ll N$. Further, let a 1 -periodic function $\varphi$ with good localization in time and frequency domain be given. For example, $\varphi$ can be chosen as tensor product of the 1-periodized $n$-dilated version $\sum_{r \in \mathbb{Z}} \tilde{\varphi}(n(x+r))$ of the

- Gaussian bell $\tilde{\varphi}(x):=\frac{1}{\pi \beta} \mathrm{e}^{-x^{2} / \beta} \quad\left(\beta:=\frac{(2 \alpha-1) m}{\alpha \pi}\right)$

- centered, cardinal $B$-spline $\tilde{\varphi}(x):=M_{2 m}(x)$ of order $2 m$

- Kaiser-Bessel function [10].

Let $\psi(x)$ denote the tensor product of the 1 -periodized, $n$-dilated version of $\tilde{\psi}(x):=\tilde{\varphi}$ $1_{[-m, m]}(x)$, where $1_{[-m, m]}$ is the characteristic function of $[-m, m]$ In case of the Gaussian bell, $\tilde{\psi}$ is simply a truncated version of $\tilde{\varphi}$, while $\tilde{\psi}$ coincides with $\tilde{\varphi}$ in the other two cases.

For $l \in I_{n}$, we-set $J_{n, m}(l):=\left\{j \in I_{M}: l-m \leq n w_{j} \leq l+m\right\}$

An algorithm for the computation of $(2.1)$ which requires only $\mathcal{O}\left((\alpha N)^{d} \log (\alpha N)+(2 m+1)^{d} M^{d}\right)$ arithmetic operations reads as follows:

Algorithm 2:1 (NFFT-Algorithm)

Input: $N, M \in \mathbb{N}, \alpha>1, n:=\alpha N, w_{j} \in \Pi^{d}, f_{j} \in \mathbb{C}\left(j \in I_{M}\right)$

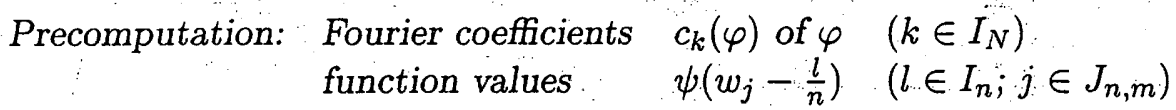

Compute 
1. $\tilde{g}_{l}:=\sum_{j \in J_{n, m}} f_{j} \psi\left(w_{j}-\frac{l}{n}\right) \quad\left(l \in I_{n}\right)$,

2. $a_{k}:=n^{-d} \sum_{l \in I_{n}} \tilde{g}_{l} \mathrm{e}^{-2 \pi i k l / n}$ by d-variate reduced $F F T$

3. $\tilde{f}_{k}:=a_{k} / c_{k}(\varphi) \quad\left(k \in I_{N}\right)$

Output: $\tilde{f}_{k}$ approximate value of $\hat{f}_{k}$

For the above functions $\varphi$ and $\alpha>1$, it was proved $[2,6,29]$ that the error $\max _{k \in I_{N}}\left|\hat{f}_{k}-\tilde{f}_{k}\right|$ decays exponentially with $m$.

We want to apply the above NFFT-algorithm for the reconstruction of Radon transformed functions. For constructive purposes, we assume that $R f$ was sampled on the grid $\mathcal{G}:=\{\boldsymbol{A} k$ : $\left.k \in \mathbb{Z}^{2}\right\}$ with

$$
A:=\left(\begin{array}{cc}
\frac{a}{M} & \frac{c}{M} \\
0 & \frac{\pi}{T}
\end{array}\right) \quad(a, c, M, T \in \mathbb{N} ; \quad a, M, T>0 ; \quad 0 \leq c<a) .
$$

Further, we assume that $a \mid M$ and $a \mid T$. Then the dual grid $\hat{\mathcal{G}}$ is generated by

$$
\hat{A}:=\left(\begin{array}{rr}
\frac{M}{a} & 0 \\
-\frac{c}{a} 2 T & 2 T
\end{array}\right)
$$

Note that we have $a=1, c=0$ for the standard grid and $a=2, c=1$ for the interlaced grid.

Remark 2.2 Indeed (2.2) is related to the more general grid (1.4) as follows: Under the additional assumption that $a_{11}, a_{12} \in \mathbb{Q}$ in $(1.4)$, there exist integers $c_{11}, c_{12}, c_{21}, c_{22}$ with $\operatorname{gcd}\left(c_{11}, c_{12}\right)=1$ and $\operatorname{gcd}\left(c_{21}, c_{22}\right)=1$ and positive numbers $T, M \in \mathbb{Q}$ such that

$$
\frac{a_{11}}{\operatorname{det}(\hat{\boldsymbol{A}})}=\frac{c_{11}}{2 T}, \frac{a_{12}}{\operatorname{det}(\hat{\boldsymbol{A}})}=\frac{c_{12}}{2 T}, \quad, \frac{a_{21}}{\operatorname{det}(\hat{\boldsymbol{A}})}=\frac{c_{21}}{M}, \frac{a_{22}}{\operatorname{det}(\hat{\boldsymbol{A}})}=\frac{c_{22}}{M}
$$

Further, there exist $k_{i} \in \mathbb{Z}^{2}$ such that $\left(c_{i 1}, c_{i 2}\right)^{\mathrm{T}} k_{i}=1(i=1,2)$. Thus, the grid $\mathcal{G}$ given by (1.4) can be alternatively generated by the matrix

$$
\left(\begin{array}{cc}
\frac{a}{M} & \frac{c}{M} \\
0 & \frac{\pi}{T}
\end{array}\right)
$$

where $a \in \mathbb{N}$ is the smallest positive integer such that $\left(\frac{a}{M}, 0\right)^{\mathrm{T}} \in \mathcal{G}$ and $c \in \mathbb{Z}$ is the smallest nonnegative integer such that $\left(\frac{c}{M}, \frac{\pi}{T}\right)^{\mathrm{T}} \in \mathcal{G}$.

For $t=-T, \ldots, T-1$, we set

$$
\varphi_{t}:=\varphi_{j, k}:=\frac{\pi}{T}(j a+k) \quad\left(k=0, \ldots, a-1 ; j=-\frac{T}{a}, \ldots, \frac{T}{a}-1\right)
$$


$\theta_{j, k}:=\left(\cos \varphi_{j, k}, \sin \varphi_{j, k}\right)^{\mathrm{T}}$ and

$$
s_{n, k}:=\frac{(k c)_{a}+n a}{M}\left(k=0, \ldots, a-1, n=-\frac{M}{a}, \cdots, \frac{M}{a}-1\right)
$$

Here $(k)_{a}$ denotes the nonnegative residue of $k$ modulo $a$. By $(2.2)$, we assume that the Radon transform $R f$ of $f$ is known at the grid points

$$
\left(s_{n, k}, \varphi_{j, k}\right) \quad\left(k=0, \ldots, a-1 ; j=0, \ldots, \frac{T}{a}-1 ; n=-\frac{M}{a}, \ldots, \frac{M}{a}-1\right) .
$$

We want to reconstruct $f$ with (1.2) on the grid

$$
\left(x_{j}, y_{k}\right)=\left(j \frac{2}{N}, k \frac{2}{N}\right) \quad\left(j, k=-\frac{N}{2}, \ldots, \frac{N}{2}-1\right)
$$

i.e. we are interested in details of size $\geq \frac{2}{N}$. Based on Shannon's sampling theorem [13], we assume that $\varepsilon_{0}(f, b)$ is small for $b \geq \frac{N}{4}$. Our Fourier reconstruction algorithm is based on the projection theorem 11 and on our NFFT-algorithm.

In the first step, we approximate the one-dimensional Fourier transforms

$$
\widehat{R f}(\sigma, \varphi)=\hat{f}(\sigma \theta)=\int_{-1}^{1} R f(s, \varphi) \mathrm{e}^{2 \pi \mathrm{i} s \sigma} \mathrm{d} s
$$

by using our sampled values $R f\left(s_{n, k}, \varphi_{j, k}\right)$ and the trapezoidal rule for the numerical integration: For $j=0, \ldots, \frac{T}{a}-1$ and $k=0, \ldots, a-1$, we obtain

$$
\begin{aligned}
\hat{g}\left(\sigma, \varphi_{j, k}\right) & :=\frac{a}{M} \sum_{n=-\frac{M}{a}}^{\frac{M}{a}-1} R f\left(s_{n, k}, \varphi_{j, k}\right) \mathrm{e}^{-2 \pi \mathrm{i}\left((k c)_{a}+n a\right) \sigma / M} \\
\hat{g}\left(\sigma, \varphi_{j, k}\right) & =\mathrm{e}^{-2 \pi \mathrm{i}(k c)_{a} \sigma / M} \frac{a}{M} \sum_{n=-\frac{M}{a}}^{\frac{M}{a}-1} R f\left(s_{n, k}, \varphi_{j, k}\right) \mathrm{e}^{-2 \pi \mathrm{i} n \sigma /\left(\frac{M}{a}\right)}
\end{aligned}
$$

and for $\sigma:=\frac{m}{\gamma}\left(m=-\frac{N \gamma}{4}, \ldots, \frac{N \gamma}{4}-1\right)$ with oversampling factor $\gamma \in \mathbb{N}$

$$
\hat{g}\left(\frac{m}{\gamma}, \varphi_{j, k}\right)=\mathrm{e}^{-2 \pi \mathrm{i}(k c)_{a} m /(\gamma M)} \frac{a}{M} \sum_{n=-\frac{M}{a}}^{\frac{M}{a}-1} R f\left(s_{n, k}, \varphi_{j, k}\right) \mathrm{e}^{-2 \pi \mathrm{in} n /\left(\frac{M \gamma}{a}\right)}
$$

Now we have for $m=u \frac{M \gamma}{a}+v \quad\left(u \in \mathbb{Z}, v \in\left\{-\frac{M \gamma}{2 a}, \ldots, \frac{M \gamma}{2 a}-1\right\}\right)$ that

$$
\begin{aligned}
\hat{g}\left(\frac{m}{\gamma}, \varphi_{j, k}\right) & =\mathrm{e}^{-2 \pi \mathrm{i}(k c)_{a} u / a} \mathrm{e}^{-2 \pi \mathrm{i}(k c)_{a} v / \gamma M} \frac{a}{M} \sum_{n=-\frac{M}{a}}^{\frac{M}{a}-1} R f\left(s_{n, k}, \varphi_{j, k}\right) \mathrm{e}^{-2 \pi \mathrm{i} n v /\left(\frac{\dot{M} \gamma}{a}\right)} \\
& =\mathrm{e}^{-2 \pi \mathrm{i}(k c)_{a} u / a} \hat{g}\left(\frac{v}{\gamma}, \varphi_{j, k}\right)
\end{aligned}
$$


Thus it suffices to evaluate $(2.5)$ for $m=-\frac{M \gamma}{2 a}, \ldots, \frac{M \gamma}{2 a}-1$. The other values follow by multiplication with complex exponentials (see $(2.6)$ ). In summary, we obtain all values $\hat{g}\left(\frac{m}{\gamma}, \varphi_{t}\right)$ by $T$ one-dimensional FFTs of length $\frac{M \gamma}{a}$ and some modulations with complex exponentials. The computational complexity can be further reduced by using the fact that for real-valued functions $f$

$$
\hat{f}(-\sigma \theta)=\overline{\hat{f}(\sigma \theta)}
$$

To improve the reconstruction quality, the first step is in general followed by a filter step. This filter step reduces the aliasing error introduced by the discretization of the Fourier integral. In our numerical computations we will apply several filters. However, our estimates of the aliasing errors in Section 3 are given without incorporation of the improvement by the filter. For this reason, we will ignore the filtering step in the following theoretical considerations.

In the second step, we approximately compute the two-dimensional inverse Fourier transform

$$
f(x)=\frac{1}{2} \int_{-\infty}^{\infty}|\sigma| \int_{-\pi}^{\pi} \hat{f}(\sigma \theta) \mathrm{e}^{2 \pi \mathrm{i} \sigma \theta x} \mathrm{~d} \varphi \mathrm{d} \sigma=\int_{-\infty}^{\infty} \sigma \int_{-\pi}^{\pi} \widehat{R f}(\sigma, \varphi) \mathrm{e}^{2 \pi \mathrm{i} \sigma \theta x} \mathrm{~d} \varphi \mathrm{d} \sigma
$$

by using our values $\hat{g}\left(\frac{m}{\gamma}, \varphi_{t}\right) \approx \hat{f}\left(\frac{m}{\gamma} \theta_{t}\right)$ and the trapezoidal rule. Based on our assumption that $\varepsilon_{0}(f, b)\left(b \geq \frac{N}{4}\right)$ is negligible small, we consider

$$
\left(f * W_{b}\right)(x)=\int_{0}^{b} \sigma \int_{-\pi}^{\pi} \hat{f}(\sigma \theta) \mathrm{e}^{2 \pi \mathrm{i} \sigma \theta x} \mathrm{~d} \varphi \mathrm{d} \sigma
$$

where

$$
\hat{W}_{b}(\xi)=1_{[-b, b]}(|\xi|) \quad\left(\xi \in \mathbb{R}^{2}\right)
$$

Note that $\hat{W}_{b}$ can be replaced by smoother functions with

$$
0 \leq \hat{W}_{b} \leq 1, \hat{W}_{b}(-\sigma)=\hat{W}_{b}(\sigma), \hat{W}_{b}(\sigma)=0 \text { for }|\sigma|>b .
$$

This leads to the filtering step mentioned before the second step of our algorithm. To simplify the notation, we omit the filtering step by a smoother function at least in our theoretical considerations.

Now discretization of the inner integral leads to

$$
\left(f * W_{b}\right)^{(\mathrm{i})}(x):=\int_{0}^{b} \sigma \frac{\pi}{T} \sum_{t=-T}^{T-1} \hat{g}\left(\sigma, \varphi_{t}\right) \mathrm{e}^{2 \pi \mathrm{i} \sigma \theta_{t} x} \mathrm{~d} \sigma
$$

and finally discretization of the outer integral with $b:=\frac{N}{4}$ to

$$
\left(f * W_{\frac{N}{4}}\right)^{(\mathrm{ii})}\left(x_{j}, y_{k}\right)=\frac{\pi}{\gamma T} \sum_{m=0}^{\frac{N \gamma}{4}} \sum_{t=-T}^{T-1} \nu_{m} \hat{g}\left(\frac{m}{\gamma}, \varphi_{t}\right) \mathrm{e}^{2 \pi \mathrm{i} m \theta_{t}\left(\begin{array}{l}
j \\
k
\end{array}\right) /\left(\frac{N \gamma}{2}\right)}
$$


where

$$
\nu_{m}= \begin{cases}\frac{1}{12} & m=0 \\ m & \text { otherwise }\end{cases}
$$

For an explanation of the choice of $\nu_{0}$ see $[21]$.

In summary, we obtain the following algorithm for the efficient computation of $\tilde{f}\left(x_{j}, y_{k}\right):=\left(f * W_{\frac{N}{4}}\right)^{(\mathrm{ii})}\left(x_{j}, y_{k}\right)$ with $\mathcal{O}\left(N^{2} \log N\right)$ arithmetic operations.

\section{Algorithm 2.3 (Fourier reconstruction algorithm)}

Input: $a, M, T \in \mathbb{N}$ and $c \in \mathbb{Z}$ grid parameters.

$R f\left(s_{n, k}, \varphi_{t}\right)\left(t=0, \ldots, T-1, k=0, \ldots, a, n=-\frac{M}{a}, \ldots, \frac{M}{a}-1\right)$.

$\gamma \in \mathbb{N}$ oversampling factor.

1. For $k=0, \ldots, a-1$ and $j=T / a, \ldots, T / a-1$ and for $m=-\frac{M \gamma}{2 a}, \ldots, \frac{M \gamma}{2 a}-1$ compute

$$
\hat{g}\left(\frac{m}{\gamma}, \varphi_{j, k}\right)=\mathrm{e}^{-2 \pi \mathrm{i}(k c)_{a} m /(\gamma M)} \frac{a}{M} \sum_{n=-\frac{M}{a}}^{\frac{M}{a}-1} R f\left(s_{n, k}, \varphi_{j, k}\right) \mathrm{e}^{-2 \pi i n m /\left(\frac{M \gamma}{a}\right)} .
$$

by $T$ one-dimensional FFTs of length $\frac{M \gamma}{a}$ and some multiplications with complex exponentials.

2. For $j, k=-\frac{N}{2}, \ldots, \frac{N}{2}-1$ compute

$$
\tilde{f}\left(x_{j}, y_{k}\right):=\frac{\pi}{\gamma T} \sum_{m=0}^{\frac{N \gamma}{4}} \sum_{t=-T}^{T-1} v_{m} \hat{g}\left(\frac{m}{\gamma}, \varphi_{t}\right) \mathrm{e}^{2 \pi i m \theta_{t}\left(\begin{array}{l}
j \\
k
\end{array}\right) /\left(\frac{N \gamma}{2}\right)}
$$

by Algorithm 2.1 with $\alpha=2$ and $d=2$.

Output: $\tilde{f}\left(x_{j}, y_{k}\right)$ approximate value of $f\left(x_{j}, y_{k}\right)$

Our Fourier reconstruction algorithm 2.3 suffers from two kinds of approximation errors: the errors introduced by applying the NFFT algorithm in step 2 and the aliasing errors arising by the discretization of the Fourier integrals. While the first kind of errors can be kept very small by an appropriate choice of the parameters of the NFFT $($ see $[24,29,10])$, the aliasing errors depend on the sampling grid $\mathcal{G}$ of the Radon transform. The relation between $\mathcal{G}$ and the introduced aliasing error will be investigated in the next section.

\section{Estimation of the aliasing error}

We are interested in the difference between $f * W_{b}$ and $\left(f * W_{b}\right)^{(\mathrm{i})}$ given by $(2.8)$ and $(2.9)$, respectively. The final discretization (2.10) also depends on the oversampling factor $\gamma$, where the discretization error can be improved by increasing $\gamma$. 
Theorem 3.1 Let $f \in C_{0}^{\infty}(\Omega)$ and let $R f \in \mathcal{S}(\mathbb{R} \times T)$ be sampled with respect to the grid generated by the matrix (2.2) with $c=1$. If $M, T \in \mathbb{N}$ satisfy one of the following conditions

i) $2 b \leq M<\frac{a}{a-1} 2 b$ and $T>\pi M(a-1) \quad(a \geq 3)$

ii) $\frac{a}{a-1} 2 b \leq M<a b$ and $T>\pi(2 a b-M) \quad(a \geq 4)$,

iii) $a b \leq M<2 a b$ and $T>\pi M \quad(a \geq 2)$

iv) $M \geq 2 a b$ and $T>2 \pi b \quad(a \geq 1)$

then

$$
\left\|f * W_{b}-\left(f * W_{b}\right)^{(i)}\right\|_{L_{\infty}(\Omega)} \leq \pi a b \varepsilon_{0}(f, b)+C\|f\|_{L_{1}(\Omega)} a \sqrt{b}\left(1-\tau^{2}\right)^{-\frac{1}{4}} e^{-\frac{2 \pi}{3} b\left(1-\tau^{2}\right)^{\frac{2}{3}}},
$$

where $C$ denotes a positive constant and

$$
\tau:= \begin{cases}\frac{\pi}{T} M(a-1) & \text { in case } \mathrm{i}) \\ \frac{\pi}{T}(2 a b-M) & \text { in case } \mathrm{ii}) \\ \frac{\pi}{T} M & \text { in case } \mathrm{ii}) \\ \frac{\pi}{T} 2 b & \text { in case } \mathrm{iv})\end{cases}
$$

To keep the notation as simple as possible we restrict our attention to $c=1$. The case $c \neq 1$ can be treated in a similar way.

Note further that our conditions i) - iv) are in agreement with Natterer's requirement $(\mathcal{A})$. See Figures 4 and 5.

Proof: Our starting point is the discretization (2.4) which is necessary in the first step of Algorithm 2.3. Regarding that $\varphi_{t}=\varphi_{j, k}$, i.e. $t:=j a+k$ and applying Poisson's summation formula, we obtain that $\hat{g}$ is related to $\widehat{R f}$ by

$$
\begin{aligned}
\hat{g}\left(\sigma, \varphi_{t}\right)= & \mathrm{e}^{-2 \pi \mathrm{i}(k c)_{a} \sigma / M} \sum_{l \in \mathbb{Z}} \widehat{R f}\left(\sigma+l \frac{M}{a}, \varphi_{t}\right) \mathrm{e}^{2 \pi \mathrm{i}\left(\sigma+l \frac{M}{a}\right) k / M} \\
= & \sum_{l \in \mathbb{Z}} \widehat{R f}\left(\sigma+l \frac{M}{a}, \varphi_{t}\right) \mathrm{e}^{2 \pi \mathrm{i} l k / a} \\
= & \sum_{r=0}^{a-1} \mathrm{e}^{2 \pi \mathrm{i} r k / a} \sum_{l \in \mathbb{Z}} \widehat{R f}\left(\sigma+r \frac{M}{a}+l M, \varphi_{t}\right) .
\end{aligned}
$$

Using (3.2) in the discretization (2.9) of the second step, we get

$$
\begin{aligned}
\left(f * W_{b}\right)^{(\mathrm{i})}(x) & =\sum_{r=0}^{a-1} \int_{0}^{b} \sigma \frac{\pi}{T} \sum_{t=-T}^{T-1}\left(\sum_{l \in \mathbb{Z}} \widehat{R f}\left(\sigma+r \frac{M}{a}+l M, \varphi_{t}\right) \mathrm{e}^{2 \pi \mathrm{i} \sigma \theta_{t} x}\right) \mathrm{e}^{2 \pi \mathrm{i} r k / a} \mathrm{~d} \sigma \\
& =\sum_{r=0}^{a-1} \int_{0}^{b} \sigma \frac{\pi}{T} \sum_{t=-T}^{T-1}\left(\sum_{l \in \mathbb{Z}} \widehat{R f}\left(\sigma+r \frac{M}{a}+l M, \varphi_{t}\right) \mathrm{e}^{2 \pi \mathrm{i} \sigma \theta_{t} x}\right) \mathrm{e}^{\mathrm{i} r 2 T \varphi_{t} / a} \mathrm{~d} \sigma
\end{aligned}
$$


This can be splitted as follows:

$$
\begin{aligned}
\left(f * W_{b}\right)^{(\mathrm{i})}(x) & =\int_{0}^{b} \sigma \frac{\pi}{T} \sum_{t=-T}^{T-1}\left(\widehat{R f}\left(\sigma, \varphi_{t}\right) \mathrm{e}^{2 \pi \mathrm{i} \sigma \theta_{t} x}\right) \mathrm{d} \sigma \\
& +\sum_{r=0}^{a-1} \int_{0}^{b} \sigma \frac{\pi}{T} \sum_{t=-T}^{T-1}\left(\sum_{t \in \mathrm{Z}} \widehat{R f}\left(\sigma+r \frac{M}{a}+l M, \varphi_{t}\right) \mathrm{e}^{2 \pi \mathrm{i} \sigma \theta_{t} x}\right) \mathrm{e}^{\mathrm{i} r 2 T \varphi_{t} / a} \mathrm{~d} \sigma \\
& +\sum_{r=1}^{a-1}\left(\int_{0}^{u(r, a)}+\int_{u(r, a)}^{b}\right) \sigma \frac{\pi}{T} \sum_{t=-T}^{T-1} \widehat{R f}\left(\sigma+r \frac{M}{a}, \varphi_{t}\right) \mathrm{e}^{2 \pi \mathrm{i} \sigma \theta_{t} x} \mathrm{e}^{\mathrm{i} r 2 T \varphi_{t} / a} \mathrm{~d} \sigma \\
& +\sum_{r=0}^{a-1}\left(\int_{0}^{o(r, a)}+\int_{o(r, a)}^{b}\right) \sigma \frac{\pi}{T} \sum_{t=-T}^{T-1} \widehat{R f}\left(\sigma-M \frac{a-r}{a}, \varphi_{t}\right) \mathrm{e}^{2 \pi \mathrm{i} \sigma \theta_{t} x} \mathrm{e}^{\mathrm{i} r 2 T \varphi_{t} / a} \mathrm{~d} \sigma,
\end{aligned}
$$

where

$$
u(r, a):= \begin{cases}b-r \frac{M}{a} & \text { if } b-r \frac{M}{a} \in(0, b] \\ 0 & \text { otherwise }\end{cases}
$$

and

$$
o(r, a):= \begin{cases}M \frac{a-r}{a}-b & \text { if } M \frac{a-r}{a}-b \in(0, b) \\ 0 \cdots & \text { if } M \frac{a-r}{a}-b \leq 0 \\ b & \text { if } M \frac{a-r}{a}-b \geq b\end{cases}
$$

By Poisson's summation formula we have for a $2 \pi$-periodic function $h$ that

$$
\frac{\pi}{T} \sum_{t=-T}^{T-1} h\left(\varphi_{t}\right) \mathrm{e}^{\mathrm{i} r 2 T \varphi_{t} / a}=\sum_{k \in \mathbb{Z}} c_{\frac{r}{a}} 2 T+k 2 T(h),
$$

where

$$
c_{k}(h):=\int_{0}^{2 \pi} h(\varphi) e^{\mathrm{i} \varphi k} \mathrm{~d} \varphi
$$

denotes ( $2 \pi$ times) the $k$-th Fourier coefficient of $h$. Now we obtain by (3.4) and Theorem 1.1 that

$$
\left(f * W_{b}\right)^{(\mathrm{i})}(x)=\left(f * W_{b}\right)(x)+e_{0}+e_{10}+e_{11}
$$

where

$$
\begin{aligned}
e_{0} & =\sum_{r=0}^{a-1} \int_{0}^{b} \sigma \frac{\pi}{T} \sum_{t=-T}^{T-1}\left(\sum_{t \in \mathbf{Z}} \widehat{R f}\left(\sigma+r \frac{M}{a}+l M, \varphi_{t}\right) \mathrm{e}^{2 \pi \mathrm{i} \sigma \theta_{t} x}\right) \mathrm{e}^{\mathrm{i} r 2 T \varphi_{t} / a} \mathrm{~d} \sigma \\
& +\sum_{r=1}^{a-1} \int_{u(r, a)}^{b} \sigma \frac{\pi}{T} \sum_{t=-T}^{T-1} \widehat{R f}\left(\sigma+r \frac{M}{a}, \varphi_{t}\right) \mathrm{e}^{2 \pi \mathrm{i} \sigma \theta_{t} x} \mathrm{e}^{\mathrm{i} r 2 T \varphi_{t} / a} \mathrm{~d} \sigma \\
& +\sum_{r=0}^{a-1} \int_{0}^{o(r, a)} \sigma \frac{\pi}{T} \sum_{t=-T}^{T-1} \widehat{R f}\left(\sigma-M \frac{a-r}{a}, \varphi_{t}\right) \mathrm{e}^{2 \pi \mathrm{i} \sigma \theta_{t} x} \mathrm{e}^{\mathrm{i} r 2 T \varphi_{t} / a} \mathrm{~d} \sigma
\end{aligned}
$$


and

$$
\begin{aligned}
e_{10} & :=\int_{0}^{b} \sigma \sum_{\substack{k \in \mathbb{Z} \\
k \neq 0}} c_{k 2 T}\left(\widehat{R f}(\sigma, \varphi) e^{2 \pi \mathrm{i} \sigma \theta x}\right) d \sigma \\
e_{11} & :=\sum_{r=1}^{a-1} \int_{0}^{u(r, a)} \sigma \frac{\pi}{T} \sum_{t=-T}^{T-1} \widehat{R f}\left(\sigma+r \frac{M}{a}, \varphi_{t}\right) \mathrm{e}^{2 \pi \mathrm{i} \sigma \theta_{t} x} \mathrm{e}^{\mathrm{i} r 2 T \varphi_{t} / a} \mathrm{~d} \sigma \\
& +\sum_{r=1}^{a-1} \int_{o(r, a)}^{b} \sigma \frac{\pi}{T} \sum_{t=-T}^{T-1} \widehat{R f}\left(\sigma-M \frac{a-r}{a}, \varphi_{t}\right) \mathrm{e}^{2 \pi \mathrm{i} \sigma \theta_{t} x} \mathrm{e}^{\mathrm{i} r 2 T \varphi_{t} / a} \mathrm{~d} \sigma .
\end{aligned}
$$

Note that $\int_{o(r, a)}^{b} \mathrm{~d} \sigma$ vanishes for $r=0$.

Let $M$ and $T$ satisfy one of the conditions i) - iv). Then we have in particular that

$$
M \geq 2 b \text { and } T>2 \pi b
$$

\section{Estimation of $e_{0}$}

Since $M \geq 2 b$ and by (2.7) the error $e_{0}$ can be estimated by

$$
\left|e_{0}\right| \leq \frac{a}{2} \frac{\pi}{T} b \sum_{t=-T}^{T-1} \int_{|\sigma| \geq b}\left|\widehat{R f}\left(\sigma, \varphi_{t}\right)\right| \mathrm{d} \sigma
$$

By Theorem 1.1 we obtain further that

$$
\left|e_{0}\right| \leq a b \pi \varepsilon_{0}(f, b)
$$

\section{Estimation of $e_{10}$}

By definition of $c_{k}(\cdot)$, we obtain

$$
e_{10}=\sum_{\substack{k \in \mathbb{Z} \\ k \neq 0}}^{b} \int_{0}^{2 \pi} \int_{0} \widehat{R f}(\sigma, \varphi) \mathrm{e}^{2 \pi \mathrm{i} \sigma \theta x} e^{\mathrm{i} \varphi k 2 T} \mathrm{~d} \varphi \mathrm{d} \sigma
$$

and further by Theorem 1.1

$$
\begin{aligned}
e_{10} & =\sum_{\substack{k \in \mathbb{Z} \\
k \neq 0}} \int_{0}^{b} \sigma \int_{0}^{2 \pi} \int_{\Omega} f(y) \mathrm{e}^{-2 \pi \mathrm{i} \sigma y \theta} \mathrm{d} y \mathrm{e}^{2 \pi \mathrm{i} \sigma \theta x} \mathrm{e}^{\mathrm{i} \varphi k 2 T} \mathrm{~d} \varphi \mathrm{d} \sigma \\
& =\sum_{\substack{k \in \mathbb{Z} \\
k \neq 0}} \int_{\Omega} f(y) \int_{0}^{b} \sigma \int_{0}^{2 \pi} \mathrm{e}^{-2 \pi \mathrm{i} \sigma(y-x) \theta} \mathrm{e}^{\mathrm{i} \varphi k 2 T} \mathrm{~d} \varphi \mathrm{d} \sigma \mathrm{d} y
\end{aligned}
$$


Set $y-x:=|y-x|(\cos \psi(x, y), \sin \psi(x, y))^{T}$ and $\alpha=\alpha(x, y):=\varphi-\psi$. Then we have that

$$
\begin{aligned}
e_{10} & =\sum_{\substack{k \in \mathbb{Z} \\
k \neq 0}} f(y) \mathrm{e}^{\mathrm{i} \psi(x, y) k 2 T} \int_{0}^{b} \sigma \int_{0}^{2 \pi} \mathrm{e}^{-2 \pi \mathrm{i} \sigma|y-x| \cos \alpha} \mathrm{e}^{\mathrm{i} \alpha k 2 T} \mathrm{~d} \alpha \mathrm{d} \sigma \mathrm{d} y \\
& =\sum_{\substack{k \in \mathbb{Z} \\
k \neq 0}} f(y) \mathrm{e}^{\mathrm{i} \psi(x, y) k 2 T} \int_{0}^{b} \sigma \frac{2 \pi}{\mathrm{i}^{2 T k}} J_{k 2 T}(2 \pi \sigma|y-x|) \mathrm{d} \sigma \mathrm{d} y
\end{aligned}
$$

Here $J_{n}$ denotes the $n$-th Bessel function of first kind

$$
J_{n}(x):=\frac{(-i)^{n}}{2 \pi} \int_{0}^{2 \pi} \mathrm{e}^{\mathrm{i} x \cos \varphi-\mathrm{i} n \varphi} \mathrm{d} \varphi \quad(n \in \mathbb{Z})
$$

The Bessel functions satisfy $J_{-n}(x)=(-1)^{n} J_{n}(x)$ and the modified Debye's formula [1, 9.3.7] [20, p.198]

$$
0 \leq J_{n}(\tau n) \leq \frac{\left(1-\tau^{2}\right)^{-\frac{1}{4}}}{(2 \pi n)^{\frac{1}{2}}} \mathrm{e}^{-\frac{n}{3}\left(1-\tau^{2}\right)^{\frac{3}{2}}} \quad(\tau \in(0,1))
$$

Since we have by (1.2) that $|y-x| \sigma \leq 2 b$ and since $T>2 \pi b$, we verify that

$$
\tau_{k}:=\frac{2 \pi \sigma|y-x|}{2 T k} \leq \frac{2 b \pi}{T}=: \tau<1 \quad(k \in \mathbb{N})
$$

Thus,

$$
\begin{aligned}
e_{10} & \leq 4 \pi\|f\|_{L_{1}(\Omega)} b \sum_{k=1}^{\infty} \frac{\left(1-\tau_{k}^{2}\right)^{-\frac{1}{4}}}{(2 \pi 2 T k)^{\frac{1}{2}}} \mathrm{e}^{-\frac{2 T k}{3}\left(1-\tau_{k}^{2}\right)^{\frac{3}{2}}} \\
& \leq C\|f\|_{L_{1}(\Omega)} \sqrt{b}\left(1-\tau^{2}\right)^{-\frac{1}{4}} \mathrm{e}^{-\frac{4 \pi b}{3}\left(1-\tau^{2}\right)^{\frac{3}{2}}}
\end{aligned}
$$

with a positive constant $C$.

\section{Estimation of $e_{11}$}

First note that $e_{11}$ only appears if $a \geq 2$, i.e. for nonstandard sampling and if $M<2 a b$. Thus, in case iv) we are done.

Let $M<2 a b$. As in the previous case, we rewrite $e_{11}$ by using Poisson's summation-formula (3.4) and Theorem 1.1 as

$$
e_{11}=\sum_{k \in \mathbb{Z}}\left(\sum_{r=1}^{a-1} \int_{\Omega} f(y)\left(I_{k, r}^{(1)}(x, y)+I_{k, r}^{(2)}(x, y)+I_{k, r}^{(3)}(x, y)\right) \mathrm{dy}\right)
$$


where

$$
\begin{aligned}
& I_{k, r}^{(1)}(x, y):=\int_{0}^{u(r, a)} \sigma \int_{0}^{2 \pi} \mathrm{e}^{\left.-2 \pi \mathrm{i} \theta\left(\left(\sigma+r \frac{M}{a}\right) y-\sigma x\right)\right)} \mathrm{e}^{\mathrm{i}\left(\frac{r}{a}+k\right) 2 T \varphi} \mathrm{d} \varphi \mathrm{d} \sigma, \\
& I_{k, r}^{(2)}(x, y):=\int_{o(r, a)}^{v(r, a)} \sigma \int_{0}^{2 \pi} \mathrm{e}^{\left.-2 \pi \mathrm{i} \theta\left(\left(\sigma-M \frac{a-r}{a}\right) y-\sigma x\right)\right)} \mathrm{e}^{\mathrm{i}\left(\frac{r}{a}+k\right) 2 T \varphi} \mathrm{d} \varphi \mathrm{d} \sigma \\
& I_{k, r}^{(3)}(x, y):=\int_{v(r, a)}^{b} \sigma \int_{0}^{2 \pi} \mathrm{e}^{-2 \pi \mathrm{i} \theta\left(\left(\sigma-M \frac{a-r}{a}\right) y-\sigma x\right)} \mathrm{e}^{\mathrm{i}\left(\frac{r}{a}+k\right) 2 T \varphi} \mathrm{d} \varphi \mathrm{d} \sigma
\end{aligned}
$$

and

$$
v(r, a):= \begin{cases}M \frac{a-r}{a} & \text { if } M \frac{a-r}{a} \in[0, b) \\ b & \text { otherwise }\end{cases}
$$

The integrals $I_{k, r}^{(1)}$ vanish if $M \geq \frac{a b}{r}$, in particular, since $M \geq 2 b$, if $r \geq \frac{a}{2}$. If $M<\frac{a b}{r}$, then we have for $\sigma \in[0, u(r, a)]$ that

$$
\rho_{r}^{(1)}(\sigma, x, y):=\left|\left(\sigma+r \frac{M}{a}\right) y-\sigma x\right| \leq 2 b-r \frac{M}{a} .
$$

The integrals $I_{k, r}^{(2)}$ vanish if $M \geq \frac{2 a b}{a-r}$. If $M<\frac{2 a b}{a-r}$, then we obtain for $\sigma \in[o(r, a), v(r, a)]$ that

$$
\rho_{r}^{(2)}(\sigma, x, y):=\left|\left(\sigma-M \frac{a-r}{a}\right) y-\sigma x\right| \leq M \frac{a-r}{a} .
$$

The integrals $I_{k, r}^{(3)}$ vanish if $M \geq \frac{a b}{a-r}$, in particular, since $M \geq 2 b$, if $r \leq \frac{a}{2}$. If $M<\frac{a b}{a-r}$, then we have for $\sigma \in[v(r, a), b]$ that

$$
\rho_{r}^{(3)}(\sigma, x, y):=\left|\left(\sigma-M \frac{a-r}{a}\right) y-\sigma x\right| \leq 2 b-M \frac{a-r}{a} .
$$

1. First we consider the nonvanishing summands in (3.10) with $1 \leq r<\frac{a}{2}(a \geq 3)$, i.e. $I_{k, r}^{(1)}$ if $M<\frac{a b}{r}$ and $I_{k, r}^{(2)}$ if $M \geq \frac{2 a b}{a-r}<4 b$. The smallest absolute value of $\frac{r}{a}+k(k \in \mathbb{Z})$ appears for $k=0$.

With respect to $I_{0, r}^{(1)}$ we see that

$$
2 \pi \rho_{r}^{(1)} \leq 2 \pi\left(2 b-r \frac{M}{a}\right)<2 T \frac{r}{a}
$$

holds true if

$$
T>\pi\left(\frac{2 a b}{r}-M\right)
$$

With respect to $I_{0, r}^{(2)}$ we have that

$$
2 \pi \rho_{r}^{(2)} \leq 2 \pi M \frac{a-r}{a}<2 T \frac{r}{a}
$$

is correct if

$$
T>\pi M\left(\frac{a}{r}-1\right)
$$


The right-hand sides of (3.11) and (3.12) increase if $r$ becomes smaller.

1.1. Let $2 b \leq M<\frac{2 a b}{a-1}$. Then $I_{k, r}^{(1)}$ and $I_{k, r}^{(2)}$ don't vanish for all $r \in \mathbb{N}, r<\frac{a}{2}$. Thus, since $M(a-1) \geq 2 a b-M$, the condition

$$
T>\pi M(a-1)
$$

ensures that $2 \pi \rho_{r}^{(\nu)}<2 T \frac{r}{a}(\nu=1,2)$ for all $r \in \mathbb{N}, r<\frac{a}{2}$.

1.2 Let $\frac{2 a b}{a-1} \leq M<a b(a \geq 4)$. Then $I_{k, 1}^{(1)}$ doesn't vanish, but $I_{k, 1}^{(2)}$ becomes zero. Since $M\left(\frac{a}{2}-1\right)<2 a b-M$, we conclude that for all nonvanishing integrals $I_{k, r}^{(\nu)}(\nu=1,2)$, the condition

$$
T>\pi(2 a b-M)
$$

ensures that $2 \pi \rho_{r}^{(\nu)}<2 T \frac{r}{a}(\nu=1,2)$ for all $r \in \mathbb{N}, r<\frac{a}{2}$.

1.3 For $M>a b$ the integrals $I_{k, r}^{(\nu)}(\nu=1,2)$ vanish.

2. Next, let $a$ be even and $r:=\frac{a}{2}(a \geq 2)$. Then the integrals $I_{k, \frac{a}{2}}^{(\nu)}(\nu=1,3)$ are zero and $I_{k, \frac{a}{2}}^{(2)}$ doesn't vanish if $M<4 b$. Here we obtain that $2 \pi \rho_{\frac{a}{2}}^{(2)} \leq 2 \pi \frac{M}{2}<2 T \frac{1}{2}$ if

$$
T>\pi M
$$

For $M<a b$, we see that $M \leq M(a-1)$ and $M<2 a b-M$ so that (3.13) and (3.14) are sharper than (3.15).

3. Finally, we consider the summands in (3.10) with $\frac{a}{2}<r \leq a-1(a \geq 3)$, i.e. $I_{k, r}^{(2)}$ doesn't vanish if $M<\frac{2 a b}{a-r}$ and $I_{k, r}^{(3)}$ if $M<\frac{a b}{a-r}$. The smallest absolute value of $\frac{r}{a}+k(k \in \mathbb{Z})$ appears for $k=-1$. With respect to $I_{-1, r}^{(2)}$ we see that

$$
2 \pi \rho_{r}^{(2)} \leq 2 \pi M \frac{a-r}{a}<2 T \frac{a-r}{a}
$$

holds true if

$$
T>\pi M
$$

With respect to $I_{-1, r}^{(3)}$ we have that

$$
2 \pi \rho_{r}^{(3)} \leq 2 \pi\left(2 b-M \frac{a-r}{a}\right)<2 T \frac{a-r}{a}
$$

is correct if

$$
T>\pi\left(\frac{2 a b}{a-r}-M\right)
$$

3.1. Let $a b \leq M<2 a b$. Then only $I_{-1, r}^{(2)}$ doesn't vanish at least for $r=a-1$ and $T$ must fulfill (3.16) to ensure that $2 \pi \rho_{r}^{(2)}<2 T\left|\frac{r}{a}+k\right|(k \in \mathbb{Z} ; r=1, \ldots, a-1)$.

3.2. Let $2 b \leq M<a b$. Then $2 a b-M>M$ and $2 a b-M \geq \frac{2 b a}{a-r}-M$ and we summarize: 
For $2 b \leq M<\frac{2 a b}{a-1}$, condition (3.13) ensures the inequality $2 \pi \rho_{r}^{(\nu)}<2 T\left|\frac{r}{a}+k\right|(k \in \mathbb{Z} ; r=$ $1, \ldots, a-1)$ for all nonvanishing integrals $I_{k, r}^{(\nu)}(\nu=1,2,3)$.

For $\frac{2 a b}{a-1} \leq M<a b$ condition (3.14) ensures the inequality $2 \pi \rho_{r}^{(\nu)}<2 T\left|\frac{r}{a}+k\right|(k \in \mathbb{Z} ; r=$ $1, \ldots, a-1)$ for all nonvanishing integrals $I_{k, r}^{(\nu)}(\nu=1,2,3)$.

Now let $\tau$ be defined by (3.1). Then we conclude similar as in the estimation of $e_{10}$ that

$$
\begin{aligned}
\left|I_{k, r}^{(1)}\right| & \leq 2 \pi \int_{0}^{u(r, a)} \sigma\left|J_{\left(\frac{r}{a}+k\right) 2 T}\left(2 \pi \rho_{r}^{(1)}(\sigma, x, y)\right)\right| \mathrm{d} \sigma \\
& \leq 2 \pi \int_{0}^{u(r, a)} \sigma \frac{\left(1-\tau^{2}\right)^{-\frac{1}{4}}}{\left(4 \pi T\left|\frac{r}{a}+k\right|\right)^{\frac{1}{2}}} \mathrm{e}^{-\left|\frac{r}{a}+k\right| 2 T\left(1-\tau^{2}\right)^{\frac{3}{2}} / 3} \mathrm{~d} \sigma \\
\left|I_{k, r}^{(2)}\right| & \leq 2 \pi \int_{o(r, a)}^{v(r, a)} \sigma\left|J_{\left(\frac{r}{a}+k\right) 2 T}\left(2 \pi \rho_{r}^{(2)}(\sigma, x, y)\right)\right| \mathrm{d} \sigma \\
& \leq 2 \pi \int_{v(r, a)} \sigma \frac{\left(1-\tau^{2}\right)^{-\frac{1}{4}}}{\left(4 \pi T\left|\frac{r}{a}+k\right|\right)^{\frac{1}{2}}} \mathrm{e}^{-\left|\frac{r}{a}+k\right| 2 T\left(1-\tau^{2}\right)^{\frac{3}{2}} / 3} \mathrm{~d} \sigma \\
\left|I_{k, r}^{(3)}\right| & \leq 2 \pi \int_{v(r, a)}^{b} \sigma\left|J_{\left(\frac{r}{a}+k\right) 2 T}\left(2 \pi \rho_{r}^{(3)}(\sigma, x, y)\right)\right| \mathrm{d} \sigma \\
& \leq 2 \pi \int_{v(r, a)}^{b} \sigma \frac{\left(1-\tau^{2}\right)^{-\frac{1}{4}}}{\left(4 \pi T\left|\frac{r}{a}+k\right|\right)^{\frac{1}{2}}} \mathrm{e}^{-\left|\frac{r}{a}+k\right| 2 T\left(1-\tau^{2}\right)^{\frac{3}{2}} / 3} \mathrm{~d} \sigma
\end{aligned}
$$

and further since $T>\pi a b$ in cases i) - iii) that

$$
\left|e_{11}\right| \leq C\|f\|_{L_{1}(\Omega)} a \sqrt{b}\left(1-\tau^{2}\right)^{-\frac{1}{4}} \mathrm{e}^{-\frac{2 \pi}{3} b\left(1-\tau^{2}\right)^{\frac{3}{2}}}
$$

Together with (3.5), (3.7) and (3.9) this yields the assertion. 

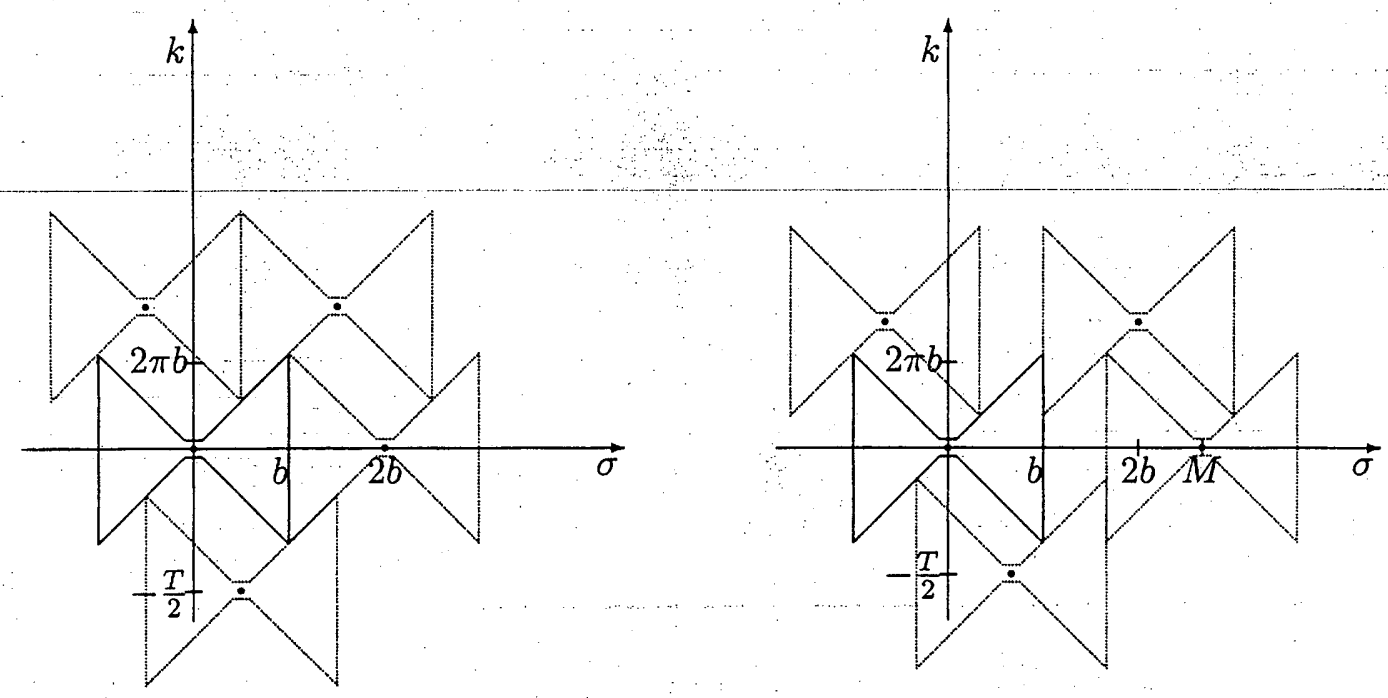

Figure 4: Dual grid (2.3) with $a=4, c=1$ and case i): $M=2 b, T=3 \frac{2 \pi b}{\mu}$ (left), case ii): $M=-\frac{4}{3} 2 b, T=\frac{8}{3} \frac{2 \pi b}{\mu}(\mu<\nu ; \nu \approx 0.95)$ (right).
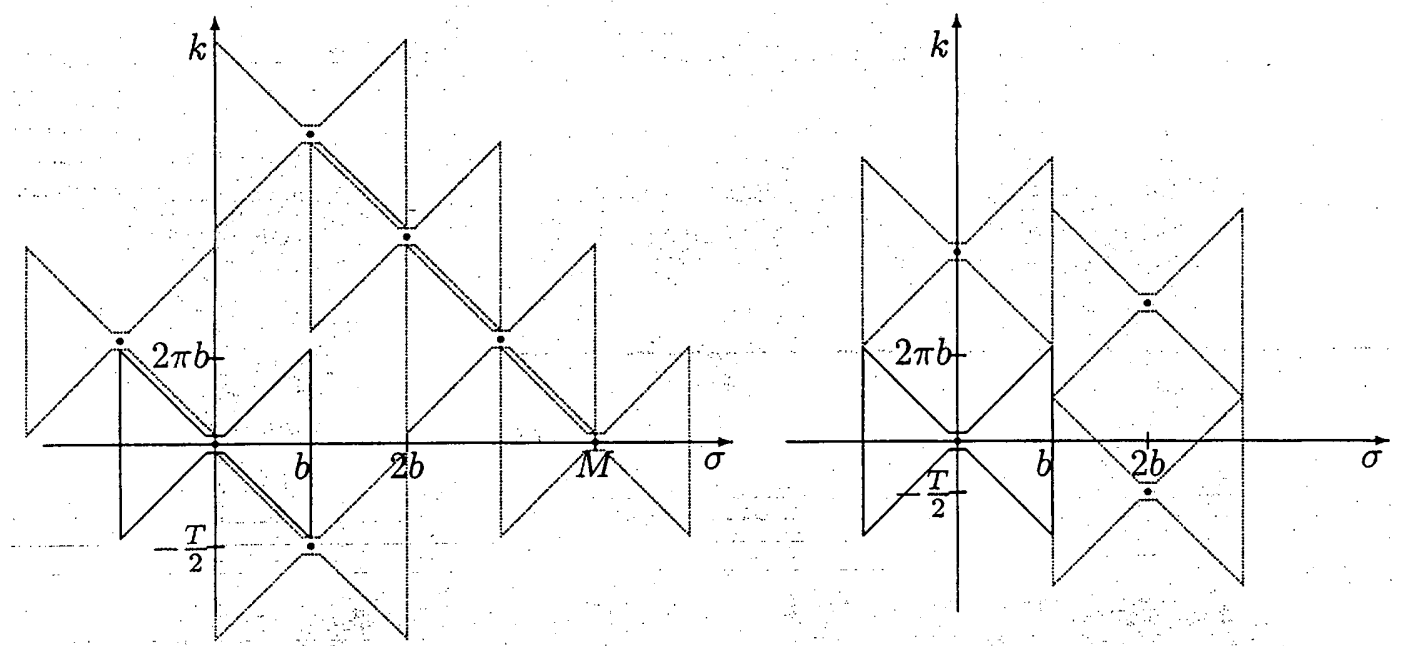

Figure 5: Dual grid (2.3) with $a=4, c=1$ and case iii): $M=4 b, T=2 \frac{2 \pi b}{\mu}$ (left), case iv): $M=8 b, T=\frac{2 \pi b}{\mu}(\mu<\nu ; \nu \approx 0.95)$ (right).

\section{Numerical Examples}

A commonly examined model in computerized tomography is the Shepp-Logan Phantom of the brain. This model consists of several ellipses so that its Radon transform can be evaluated analytically. In order to get a sampled version of the phantom and its Radon transform we have use the software packages "RadonAna" [30]. The original image (Figure 6.(left)) is of size 
$N \times N=256 \times 256$ and its sinogram of size $R \times T=256 \times 600$. Figure 6 (right) presents the reconstructed image obtained by the filtered backprojection. Here we have used the software package "iradon" [30].
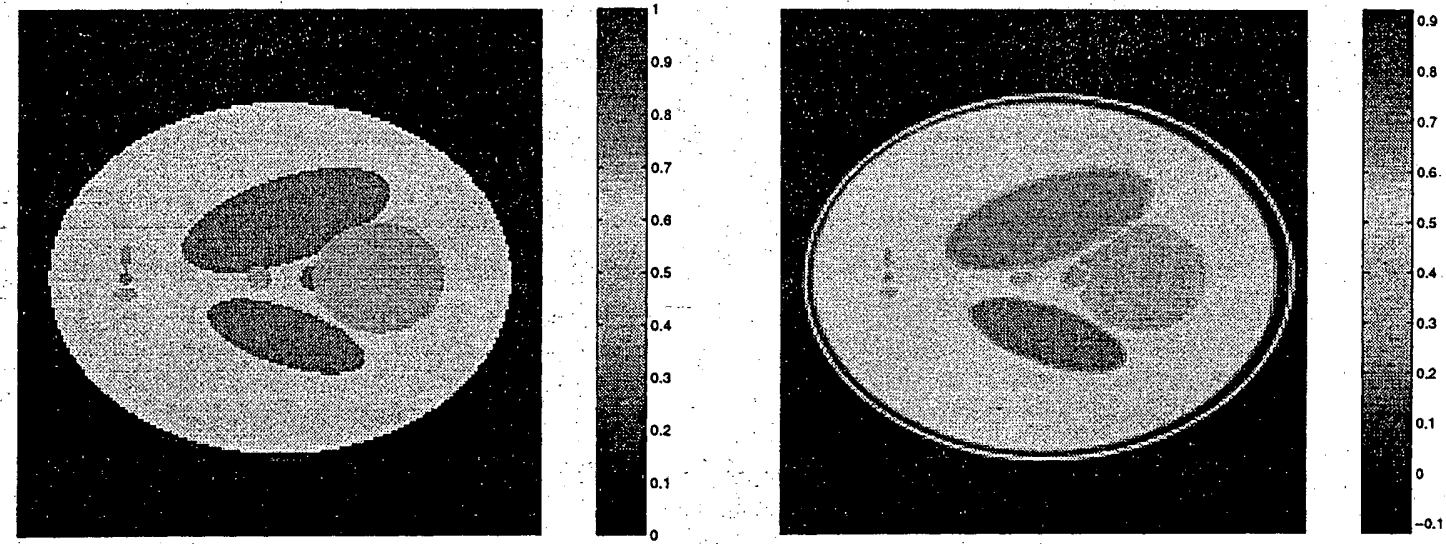

Figure 6: Shepp-Logan phantom, original (left), reconstructed image by filtered backprojection (right).

Our Fourier reconstruction algorithms were implemented in C on a SGI O2. The reconstructed images in Figure 7 were computed by Algorithm 2.3 with $a=1, c=0$, i.e with respect to the standard grid (left) and with $a=2, c=1$, i.e. with respect to the interlaced grid (right). Figure 7 shows the reconstructed image with $a=4, c=1$. All examples were computed with oversampling factor $\gamma=2$. Further, we have used the filter $\operatorname{sinc}^{2}$ for Figure 7 and the filter $\operatorname{sinc}^{4}$ for Figure 8 .

We have computed Step 2 of Algorithm 2.3 by applying Algorithm 2.1 with $d=2$, oversampling factor $\alpha=2$, the tensor product of a dilated periodized Gaussian bell $\varphi$ and the tensor product of its truncated version $\psi$, where $m=3$ and $\beta=\frac{2 \alpha m}{(2 \alpha-1) \pi}=\frac{4}{\pi}$.
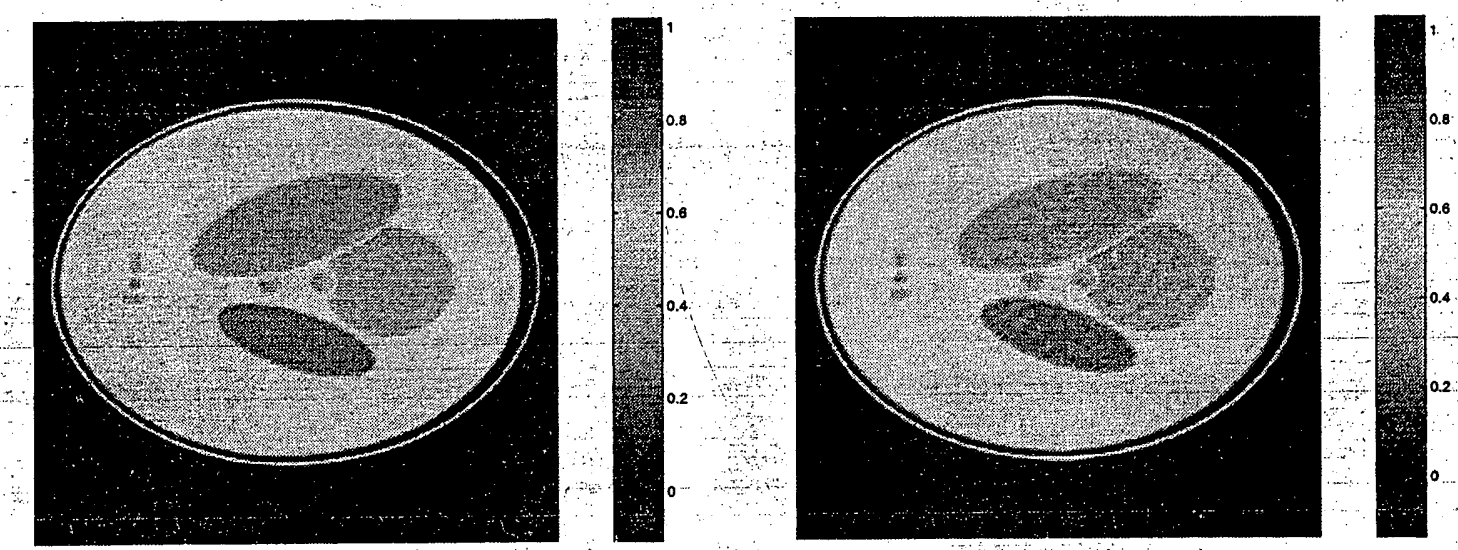

Figure 7: Reconstructed image by Algorithm 2.3 with standard grid (left) and with interlaced grid (right). 


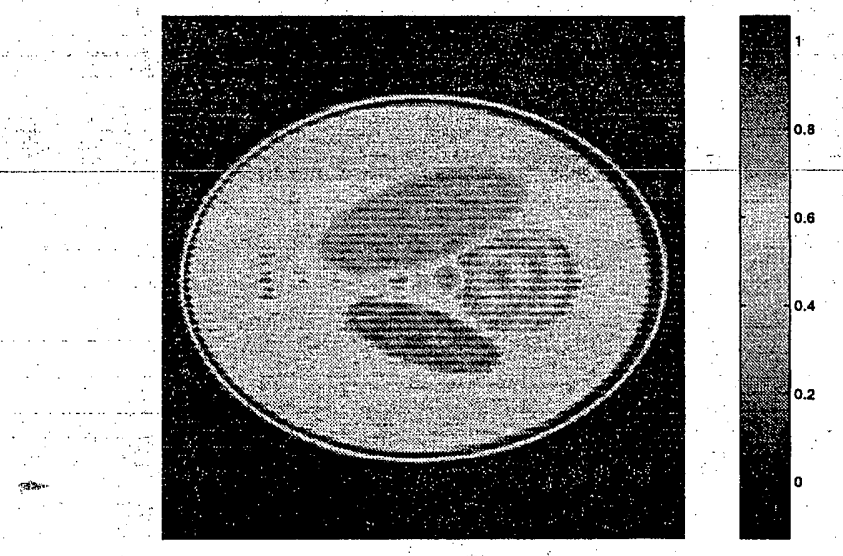

Figure 8: Reconstructed image by Algorithm 2.3 with $a=4$ and $c=1$.

Finally, note that we can detect differences in the quality of the reconstructed images much better if we are given colored images. For this we refer to

http://www.math.mu-luebeck.de/potts/radon/interl.html.

\section{References}

[1] M. Abramowitz and I. A. Stegun. Handbook of Mathematical Functions. Dover, New York 1970.

[2] G. Beylkin. On the fast Fourier transform of functions with singularities. Appl. Comput. Harmon. Anal., 2:363 - 381, 1995.

[3] R. N. Bracewell and A. C. Riddle. Strip integration in radio astronomy. Australian J. Phys., 9:198-217, 1956 .

[4] A. M. Cormack. Sampling the Radon transform with beams of finite width. Phys. Med. Biol., $23: 1141$ - 1148, 1978.

[5] A. J. W. Duijndam and A. M. Schonewille. Nonuniform fast Fourier transform. Preprint, 1997.

[6] A. Dutt and V. Rokhlin. Fast Fourier transforms for nonequispaced data. SIAM J. Sci. Statist Comput., 14:1368 - 1393, 1993 .

[7] P. Edholm and G. Herman. Linograms in image reconstruction from projections. IEEE Trans. Med. Imag., 6:301 - 307, 1987.

[8] B. Elbel Mehrdimensionale Fouriertransformation für nichtäquidistante Daten. Master's thesis, Technical University Darmstadt, 1998.

[9] B. Elbel and G. Steidl. Fast Fourier transform for nonequispaced data. In C. K. Chui and L. L. Schumaker, editors, Approximation Theory IX, Vanderbilt University Press, Nashville, 1998. 
[10] K. Fourmont. Schnelle Fourier-Transformation bei nichtäquidistanten Gittern und tomographische Anwendungen. $\mathrm{PhD}$ thesis, University of Münster, 1999.

[11] D. Gottlieb, B. Gustafsson and P. Forssen. On the direct Fourier method for computer tomography. IEEE Trans. Med. Imag., 9:223 - 232, 2000.

[12] J. I. Jackson. Selection of a convolution function for Fourier inversion using gridding. IEEE Trans. Medical Imaging, 10:473 - 478, 1991.

[13] A. J. Jerry. The Shannon sampling theorem - its various extensions and applications: a tutorial review. Proc. IEEE, 1965.

[14] W. Klaverkamp. Tomographische Bildreconstruction mit direkten algebraischen Verfahren. PhD thesis, University Münster, 1991.

[15] H. Kruse. Resolution of Reconstruction Methods in Computerized Tomography. SIAM J. Sci. Statist. Comput., 10:447 - 474, 1989.

. [16] M. Kaveh and M. Soumekh. Computer-assisted diffraction tomography. In H. Stark, editor, Image Recovery: Theory and Applications, pages 369-413, Academic Press, Orlando, 1987.

[17] M. Kaveh, M. Soumekh, and J. Greenleaf. Signal processing for diffraction tomography. IEEE Transactions on Sonics and Ultrasonics, SU-31, 1984.

[18] A. G. Lindgren and P. A. Rattey. The inverse discrete Radon transform with application to tomographic imaging using projection data. Adv. in Electronics and Electron. Phys., $56: 359-410,1981$.

[19] F. Natterer. Fourier reconstruction in tomography. Numer. Math., 47:343 - 353, 1985.

[20] F. Natterer. The Mathematics of Computerized Tomography. Teubner, Stuttgart, Chichester, 1986.

[21] F. Natterer and F. Wübbeling. Mathematical Methods in Image Reconstruction. 2000.

-[22] J. O'Sullivan. A fast sinc function gridding algorithm for Fourier inversion in computer tomography. IEEE Trans. Med. Imag., 4:200 - 207, 1985.

[23] J. Pelt. Fast computation of trigonometric sums with application to frequency analysis of astronomical data. Preprint, University of Delft, 1998.

[24] D. Potts, G. Steidl, and M. Tasche. Fast Fourier transforms for nonequispaced data. A tutorial. In J. J. Benedetto and P. Ferreira, editors, Modern Sampling Theory: Mathematics and Application, chapter 12, pages 253 - 274. Birkhäuser, 2000.

[25] D. Potts and G. Steidl. New Fourier reconstruction algorithms for computerized tomography. Proc. SPIE Conf., San Diego, 2000.

[26] H. Schomberg and J. Timmer. The gridding method for image reconstruction by Fourier transformation. IEEE Trans. Med. Imag., MI-14:596 - 607, 1995.

[27] J. Schulte. Fourier-Rekonstruktionen in der Computer-Tomographie. Master's thesis, University of Münster, 1994. 
[28] R. A. Scramek and F. R. Schwab. Imaging. In F. R. S. R. Perley and A. Bridle, editors, Astronomical Society of the Pacific Conference, Vol 6, pages 117-138. 1988.

[29] G. Steidl. A note on fast Fourier transforms for nonequispaced grids. Adv. Comp. Math, 9:337 - 352, 1998 .

[30] P. Toft. The Radon Transform - Theory and Implementation. PhD thesis, Technical University of Denmark, 1996.

[31] J. Walden. Analysis of the direct Fourier method for computer tomography. IEEE Trans. Med. Imag., 9:211 - 222, 2000.

[32] A. Ware. Fast approximate Fourier transforms for irregularly spaced data. SIAM Review, 40:838 - 859, 1998. 